\title{
ESTRUTURA DA ASSEMBLEIA DE SCOLYTINAE (COLEOPTERA: CURCULIONIDAE) EM ÁREAS FLORESTADAS COM Eucalyptus spp. NO SUL DO RIO GRANDE DO SUL
}

\author{
SCOLYTINAE ASSEMBLAGE STRUCTURE (COLEOPTERA: CURCULIONIDAE) IN FORESTED \\ AREAS WITH Eucalyptus spp. IN SOUTHERN RIO GRANDE DO SUL STATE
}

\author{
Jutiane Wollmann ${ }^{1}$ Mauro Silveira Garcia ${ }^{2}$ Carlos Alberto Hector Flechtmann ${ }^{3}$ Elder Finkenauer ${ }^{4}$ \\ Flávio Roberto Mello Garcia ${ }^{5}$
}

\section{RESUMO}

O objetivo deste trabalho foi contribuir para o conhecimento da assembleia de besouros Scolytinae associada a plantios de eucalipto no sul do Estado do Rio Grande do Sul, Brasil e determinar espécies de importância à cultura. Amostras quinzenais foram retiradas de cinco fazendas da Fibria Celulose S/A, florestadas com Eucalyptus spp., no período de fevereiro de 2006 a dezembro de 2010, com armadilhas de interceptação de voo iscadas com etanol a 95\%. Foram coletados 7.365 exemplares em 76 espécies, 18 gêneros e sete tribos. Corthylini, representada pelos gêneros Corthylus, Monarthum, Amphicranus, Metacorthylus, Microcorthylus e Tricolus e Xyleborini, por Ambrosiodmus, Dryocoetoides, Xyleborinus, Xyleborus e Xylosandrus foram as tribos mais representativas, com $42,1 \%$ e 31,6\% espécies, respectivamente. Xylosandrus retusus e Xyleborinus saxeseni foram as espécies mais abundantes com 52,64\% e 27,98\% do total de espécimes amostradas, seguida por Corthylus antennarius, com 4,15\% besouros coletados. Quinze espécies foram comuns a todos os locais, incluindo Xyleborus ferrugineus, considerada praga desta cultura, enquanto 25 espécies foram encontradas exclusivamente em apenas um local. A fazenda Cerro Alegre apresentou a maior riqueza de espécies e maior quantidade de espécies exclusivas; São Manoel demonstrou o maior índice de diversidade e equitabilidade; Aroeira teve a maior frequência de Scolytinae e São Francisco evidenciou a maior abundância e dominância. O estudo das assembleias de Scolytinae evidenciou o predomínio das espécies Xylosandrus retusus e Xyleborinus saxeseni em todos os ambientes estudados.

Palavras-chave: besouros-da-ambrosia; análise faunística; diversidade; eucalipto.

\section{ABSTRACT}

The aim of this study was to contribute to the knowledge of the assembly of Scolytinae beetles associated with Eucalyptus plantations in southern Rio Grande do Sul state, Brazil, and determine species of importance to the culture. Biweekly samples were taken from five Fibria Celulose S/A farms forested with Eucalyptus spp., from February 2006 to December 2010, with flight intercept traps baited with 95\% ethanol. We collected 7,365 specimens within 76 species, 18 genera, and seven tribes. Corthylini, represented by the

1 Engenheira Agrônoma, Doutoranda em Fitossanidade, Faculdade de Agronomia Eliseu Maciel, Universidade Federal de Pelotas, Av. Eliseu Maciel, s/n, Campus Universitário, CEP 96010-900, Pelotas (RS), Brasil. jutianewollmann@hotmail.com

2 Engenheiro Agrônomo, Dr., Professor do Departamento de Fitossanidade, Faculdade de Agronomia Eliseu Maciel, Universidade Federal de Pelotas, Av. Eliseu Maciel, s/n, Campus Universitário, CEP 96010-900, Pelotas (RS), Brasil. garciasmauro@yahoo.com.br

3 Engenheiro Agrônomo, Dr., Professor Adjunto do Departamento de Fitossanidade, Engenharia Rural e Solos, Faculdade de Engenharia, Universidade Estadual Paulista Júlio de Mesquita Filho, Av. Brasil, 56, Campus de Ilha Solteira, CEP 15385-000, Ilha Solteira (SP), Brasil. flechtma@bio.feis.unesp.br

4 Ecólogo, Coordenador na empresa Equilíbrio Proteção Florestal, R. Odonaldo Ferreira Dutra, 353, CEP 79621-130, Três Lagoas (MS), Brasil. elder.finkenauer@yahoo.com.br

5 Biólogo, Dr., Professor do Departamento de Ecologia, Zoologia e Genética, Instituto de Biologia, Universidade Federal de Pelotas, Av. Eliseu Maciel, s/n, Campus Universitário, CEP 96010-900, Pelotas (RS), Brasil. flaviormg@ hotmail.com

Recebido para publicação em 25/02/2014 e aceito em 15/08/2016 
genera Corthylus, Monarthum, Amphicranus, Metacorthylus, Microcorthylusand Tricolus, and Xyleborini, by Ambrosiodmus, Dryocoetoides, Xyleborinus, Xyleborus and Xylosandrus, were the most representative tribes, with $42.1 \%$ and $31.6 \%$ species, respectively. Xylosandrus retusus (Eichhoff) and Xyleborinus saxeseni (Ratzeburg) were the most abundant species, with $52.64 \%$ and $27.98 \%$ of all specimens sampled, followed by Corthylus antennarius Schedl with $4.15 \%$ beetles collected. Fifteen species were common to all sites, including Xyleborus ferrugineus (Fabricius), considered a potential pest to the culture, while 25 species were found exclusively in only one site. The farm Cerro Alegre had the highest species richness and larger number of exclusive species; São Manoel farm presented the highest diversity index and equitability; Aroeira farm had the highest frequency of Scolytinae and São Francisco farm showed the greatest abundance and dominance. The study of Scolytinae assemblages showed the predominance of Xylosandrus retusus and Xyleborinus saxeseni in all studied environmentals.

Keywords: ambrosia beetles; faunistic analysis; diversity; eucalypt.

\section{INTRODUÇÃO}

Atualmente, no Brasil, os plantios comerciais de Eucalyptus spp. correspondem a aproximadamente 5,1 milhões de hectares, onde cerca de 284 mil concentram-se no Rio Grande do Sul (ASSOCIAÇÃO BRASILEIRA DE PRODUTORES DE FLORESTAS PLANTADAS, 2013) sobre o Bioma Pampa (SILVA, 2012). Este bioma se caracteriza por uma complexa vegetação, na qual predominam os campos nativos, entremeados por capões de mata, matas ciliares, formações arbustivas, afloramentos rochosos e banhados (BRASIL, 2007).

A expansão de monoculturas em detrimento à vegetação natural diminui cada vez mais a diversidade do habitat local, no qual a modificação de comunidades vegetais fica sujeita a danos causados por pragas (ALTIERI; LETOURNEAU, 1984). Isto se deve ao fato de que a estrutura original da vegetação pode determinar a distribuição espacial e a disponibilidade de recursos para os insetos (MORAES et al., 2002).

Besouros-da-casca e besouros-da-ambrosia (Curculionidae: Scolytinae e Platyponinae) estão entre os grupos de insetos mais prejudiciais às florestas (CIESLA, 2011), tanto pelo dano causado quanto pela dificuldade de controle (BERTI FILHO, 1979). Besouros-da-ambrosia são menos específicos em sua seleção hospedeira do que os da casca, visto que estes últimos se alimentam de tecidos da planta (fleófagos) e os primeiros de fungos (xilomicetófagos), que são introduzidos no hospedeiro durante a construção de suas galerias (HARRINGTON, 2005; WOOD, 1982).

Dentre os Scolytinae, os besouros-da-ambrosia são o grupo dominante no Brasil, sendo que a maioria das espécies pertence à tribo Xyleborini (FLECHTMANN et al., 1995), que se destaca como a mais significativa e rica em espécies e a mais invasiva de todos os besouros-da-ambrosia (HULCR; SMITH, 2010). Perdas econômicas causadas por estes besouros estão diretamente relacionadas à degradação de madeira recém-cortada (BEAVER, 1987). Todavia, árvores sadias também podem ser atacadas por espécies do gênero Xyleborus, que podem broquear qualquer parte da planta hospedeira (WOOD, 1982). Em Em besouros-da-casca as perdas são devidas, principalmente, à morte de árvores (OKLAND et al., 2011).

Desde o início dos levantamentos, na década de 1970, tem sido observado um aumento em abundância e riqueza de Scolytinae em florestas implantadas (FLECHTMANN et al., 1995) e, desde então, inúmeros estudos têm sido conduzidos para conhecer a fauna destes besouros. Entretanto, ainda há pouca informação associando espécies de Scolytinae e seus hospedeiros no Brasil (OLIVEIRA; FLECHTMANN; FRIZZAS, 2008), o que torna difícil estimar o real dano que causam em plantios florestais e a adoção de medidas de controle (FLECHTMANN; OTTATI; BERISFORD, 2001).

O objetivo deste estudo foi caracterizar a estrutura da assembleia de Scolytinae associada a plantios de Eucalyptus spp. em cinco áreas inseridas no Bioma Pampa, Rio Grande do Sul e identificar as espécies importantes a esta cultura.

\section{MATERIAL E MÉTODOS}

Os coleópteros foram amostrados de fevereiro de 2006 a outubro de 2010 em cinco áreas 
pertencentes à empresa Fibria Celulose S/A florestadas com Eucalyptus dunnii, Eucalyptus globulus, Eucalyptus saligna e alguns materiais clonais de E. grandis $x$ E. urophylla, com aproximadamente dois anos de idade (Tabela 1).

TABELA 1: Áreas de coleta de Scolytinae no sul do Estado do Rio Grande do Sul. Localização e características dos cultivos. Fevereiro de 2006 a outubro de 2010.

TABLE 1: Scolytinae collection areas in the southern Rio Grande do Sul, Brazil. Location and characteristics of crops. February 2006 to October 2010.

\begin{tabular}{cccccc}
\hline Localidades & Localização & $\begin{array}{c}\text { Área florestada } \\
\text { (hectare) }\end{array}$ & $\begin{array}{c}\text { Área total } \\
\text { (hectare) }\end{array}$ & Área preservada (\%) & $\begin{array}{c}\text { Material } \\
\text { genético }\end{array}$ \\
\hline Santa Rosa (A. Grande) & $\begin{array}{l}32^{\circ} 06^{\prime} 31^{\prime \prime} \mathrm{S} \\
53^{\circ} 07^{\prime} 19^{\prime \prime} \mathrm{W}\end{array}$ & 373,11 & $1.038,14$ & 58,97 & 3,6 \\
& $\begin{array}{l}31^{\circ} 28^{\prime} 41^{\prime \prime} \mathrm{S} \\
53^{\circ} 40^{\prime} 49^{\prime \prime} \mathrm{W}\end{array}$ & $1.456,26$ & $4.090,15$ & 40,68 & 7,8 \\
Aroeira (Candiota) & $\begin{array}{l}31^{\circ} 54^{\prime} 06^{\prime \prime} \mathrm{S} \\
53^{\circ} 38^{\prime} 13^{\prime \prime} \mathrm{W}\end{array}$ & $1.600,45$ & $2.846,57$ & 42,23 & 3 \\
São Francisco (P. Altas) & $\begin{array}{l}31^{\circ} 33^{\prime} 42^{\prime \prime} \mathrm{S} \\
53^{\circ} 26^{\prime} 07^{\prime \prime} \mathrm{W}\end{array}$ & 251,77 & 631,53 & 58,56 & $1,4,5$ \\
São Manoel (P. Machado) & & & & 2,3 \\
Cerro Alegre (Piratini) & $\begin{array}{l}31^{\circ} 19^{\prime} 59^{\prime \prime} \mathrm{S} \\
53^{\circ} 18^{\prime} 37^{\prime \prime} \mathrm{W}\end{array}$ & $1.107,43$ & $3.786,00$ & 68,52 & 2,3 \\
\hline
\end{tabular}

Em que: Material genético: $1=E$. dunnii; $2=E$. globulus; $3=E$. saligna; Híbridos de E. grandis $x$ E. urophylla: $4=\mathrm{P} 4295 \mathrm{H} ; 5=\mathrm{C} 186 \mathrm{H} ; 6=\mathrm{AE} ; 7=\mathrm{TC} 31 \mathrm{H} ; 8=\mathrm{VR} 3748 \mathrm{H}$.

As áreas em que o estudo foi conduzido foram: Fazenda Santa Rosa, município de Arroio Grande; Fazenda São Manoel, Pinheiro Machado; Fazenda São Francisco, Pedras Altas; Fazenda Cerro Alegre, Piratini; e Fazenda Aroeira, Candiota, localizadas na região fisiográfica da Campanha e da Serra do Sudeste, cuja vegetação é formada por Estepe e Floresta Estacional Semidecidual (BRASIL, 2007) e clima tipo Cfa de Köppen, que se caracteriza pelo clima temperado úmido (KOTTEK, 2006). A vegetação adjacente aos plantios, que compreende o restante de cada área em diferentes proporções, é constituída por áreas de preservação permanente, como banhados/brejos, mata ciliar, campo nativo, afloramentos rochosos e áreas destinadas à reserva legal.

Para a realização das coletas, em cada uma das áreas foram instaladas três armadilhas de intercepção de voo modelo ESALQ-84 (BERTI FILHO; FLECHTMANN, 1986) a 1,5 m de altura do solo, que corresponde ao padrão máximo de voo geralmente apresentado por besouros-da-ambrosia (TURNBOUW; FRANKLIN, 1980; ATKINSON; FOLTZ; CONNOR, 1988) tendo como referência o cone de captura (FLECHTMANN et al., 1995) e iscadas com álcool comercial (95\%) para a atração dos insetos. As armadilhas foram instaladas nos materiais genéticos disponíveis para estudos nas fazendas (Tabela 1), posicionadas a $50 \mathrm{~m}$ da borda em direção ao interior dos talhões e distantes umas das outras em aproximadamente $200 \mathrm{~m}$. As coletas foram realizadas quinzenalmente, quando os insetos capturados eram retirados do frasco coletor e o álcool renovado. Os insetos coletados foram triados e armazenados no Laboratório de Biologia de Insetos e Controle Biológico do Departamento de Fitossanidade da Faculdade de Agronomia Eliseu Maciel, Universidade Federal de Pelotas, RS, onde foram armazenados em freezer para futura triagem e identificação em nível específico por taxonomista no grupo.

A análise faunística dos dados foi realizada pelo programa ANAFAU (MORAES et al., 2003), no qual foram determinados os índices de abundância, frequência, dominância (método de Laroca e Mielke), constância e diversidade de Shannon-Wiener (H'). 


\section{RESULTADOS E DISCUSSÃO}

Nas cinco áreas amostradas foram coletados 7.365 espécimes de Scolytinae pertencentes a sete tribos, 18 gêneros e 76 espécies. A maior riqueza foi encontrada na fazenda Cerro Alegre, representando $69,7 \%(\mathrm{~S}=53)$ do total de espécies coletadas, seguida por Santa Rosa, com 52,6\% ( $\mathrm{S}=40)$, São Manoel, $50 \%(\mathrm{~S}=38)$, São Francisco, $46 \%(\mathrm{~S}=35)$ e Aroeira, $40,8 \%(\mathrm{~S}=31)$ (Tabela 2$)$.

TABELA 2: Riqueza de espécies de Scolytinae em fazendas de Eucalyptus spp no sul do Rio Grande do Sul, Brasil. Fevereiro de 2006 a outubro de 2010.

TABLE 2: Species richness of Scolytinae in farms of Eucalyptus spp., in southern Rio Grande do Sul state, Brazil. February 2006 to October 2010.

\begin{tabular}{|c|c|c|c|c|c|}
\hline Espécies & Aroeira (Candiota) & $\begin{array}{l}\text { Cerro Alegre } \\
\text { (Piratini) }\end{array}$ & $\begin{array}{l}\text { Santa Rosa } \\
\text { (A.Grande) }\end{array}$ & $\begin{array}{l}\text { São Manoel } \\
\text { (P.Machado) }\end{array}$ & $\begin{array}{c}\text { São Francisco } \\
\text { (P.Altas) }\end{array}$ \\
\hline XILOMICETÓFAGAS & & & & & \\
\hline Ambrosiodmus hagedorni (Iglesias, 1914) & - & 2 & 2 & - & - \\
\hline Ambrosiodmus obliquus (Le Conte, 1878) & 4 & 3 & - & 2 & - \\
\hline Ambrosiodmus rusticus (Wood, 1974) & - & 1 & - & - & - \\
\hline Ambrosiodmus sp.1 & 4 & 5 & 2 & 2 & 4 \\
\hline Ambrosiodmus sp.2 & 1 & 1 & - & - & - \\
\hline Amphicranus sp.1 & - & 1 & 1 & 1 & - \\
\hline Amphicranus sp. 2 & 1 & 1 & 3 & 1 & - \\
\hline Amphicranus sp. 3 & - & - & 3 & - & - \\
\hline Coptoborus gracilens Wood, 2007 & - & - & - & 3 & - \\
\hline Corthylus antennarius Schedl, 1966 & 38 & 69 & 76 & 74 & 49 \\
\hline Corthylus comitabilis Wood, 2007 & - & - & - & 2 & - \\
\hline Corthylus punctatus Eggers, 1943 & 1 & 7 & 8 & 6 & 14 \\
\hline Corthylus sp.1 & 3 & 2 & 7 & 1 & 3 \\
\hline Corthylus sp.2 & - & 6 & 1 & 6 & 1 \\
\hline Corthylus sp. 3 & - & 1 & - & 1 & 2 \\
\hline Corthylus sp.4 & 2 & 3 & 5 & 4 & 5 \\
\hline Corthylus sp.5 & - & 2 & - & - & - \\
\hline Corthylus sp.6 & 2 & 2 & - & - & - \\
\hline Corthylus sp.7 & - & 3 & 1 & 11 & - \\
\hline Corthylus sp.8 & 1 & - & - & - & 1 \\
\hline Corthylus sp.9 & 1 & - & - & - & - \\
\hline Corthylus sp.10 & - & - & - & 2 & - \\
\hline Cryptocarenus heveae (Hagedorni, 1912) & - & - & - & 1 & - \\
\hline Cryptocarenus brevicollis Eggers, 1937 & - & - & 1 & 2 & - \\
\hline Cryptocarenus diadematus Eggers, 1937 & - & 1 & - & 1 & 1 \\
\hline Cryptocarenus seriatus Eggers, 1933 & - & 4 & - & 1 & 2 \\
\hline Cryptocarenus sp.1 & - & - & - & 1 & - \\
\hline Dryocoetoides asperulus (Eggers, 1931) & - & - & - & 1 & - \\
\hline Hypothenemus dolosus Wood, 1974 & - & - & - & 1 & - \\
\hline Hypothenemus eruditus Westwood, 1836 & - & 2 & 4 & - & 12 \\
\hline Hypothenemus sp.1 & - & - & 3 & - & 3 \\
\hline Hypothenemus sp.2 & 3 & - & 3 & - & 2 \\
\hline Hypothenemus sp. 3 & 1 & 1 & 6 & - & 1 \\
\hline Hypothenemus sp.4 & - & - & - & - & 13 \\
\hline Metacorthylus sp.1 & 1 & - & 5 & - & - \\
\hline Microcorthylus quadridens Wood, 2007 & 5 & 4 & 17 & 8 & 6 \\
\hline Microcorthylus sp.1 & 1 & - & 1 & - & - \\
\hline Monarthrum cristatum Wood \& Bright, 1992 & - & 3 & - & 4 & - \\
\hline Monarthrum sp.1 & 4 & 1 & 4 & - & 5 \\
\hline Monarthrum sp. 2 & - & - & - & 1 & 1 \\
\hline Monarthrum sp. 3 & 5 & 79 & 22 & 15 & 9 \\
\hline Monarthrum sp.4 & 8 & 42 & 20 & 3 & 2 \\
\hline Monarthrum sp.5 & 4 & 31 & 22 & 14 & 1 \\
\hline
\end{tabular}


TABELA 2: Continuação...

TABLE 2: Continued...

\begin{tabular}{|c|c|c|c|c|c|}
\hline Espécies & Aroeira (Candiota) & $\begin{array}{l}\text { Cerro Alegre } \\
\text { (Piratini) }\end{array}$ & $\begin{array}{l}\text { Santa Rosa } \\
\text { (A.Grande) }\end{array}$ & $\begin{array}{l}\text { São Manoel } \\
\text { (P.Machado) }\end{array}$ & $\begin{array}{l}\text { São Francisco } \\
\text { (P.Altas) }\end{array}$ \\
\hline Monarthrum sp.6 & 1 & 21 & 3 & - & - \\
\hline Monarthrum sp.7 & - & 9 & 1 & - & 2 \\
\hline Monarthrum sp.8 & - & 25 & 7 & - & 1 \\
\hline Monarthrum sp.9 & - & 3 & 1 & 2 & - \\
\hline Monarthrum sp.10 & - & 3 & 2 & 1 & - \\
\hline Monarthrum sp.11 & - & 1 & 1 & - & - \\
\hline Scolytodes bolivianus Eggers, 1928 & - & 1 & - & - & - \\
\hline Tricolus sp.1 & - & 2 & 1 & - & 6 \\
\hline Xyleborinus gracilis (Eichhoff, 1868) & - & 5 & - & 3 & - \\
\hline Xyleborinus saxeseni (Ratzeburg, 1837) & 734 & 20 & 124 & 17 & 1166 \\
\hline Xyleborinus sentosus (Eichhoff, 1868) & 5 & 3 & 7 & 3 & 23 \\
\hline Xyleborinus sp.1 & - & - & - & 8 & - \\
\hline Xyleborinus sp. 2 & 56 & 14 & 7 & 1 & 51 \\
\hline Xyleborinus sp.3 & - & 1 & - & - & 6 \\
\hline Xyleborinus sp.4 & - & 1 & - & - & - \\
\hline Xyleborinus sp. 5 & - & 4 & - & - & - \\
\hline Xyleborus affinis Eichhoff, 1867 & 1 & 3 & 17 & - & - \\
\hline Xyleborus ferrugineus (Fabricius, 1801) & 9 & 61 & 40 & 34 & 25 \\
\hline Xyleborus neivai Eggers, 1928 & 5 & 8 & 3 & 3 & 1 \\
\hline Xyleborus reconditus Schedl, 1963 & - & - & - & - & 1 \\
\hline Xyleborus sp. 1 & 1 & 1 & - & - & 1 \\
\hline Xyleborus sp.2 & - & - & - & - & 6 \\
\hline Xyleborus sp. 3 & - & - & 1 & - & - \\
\hline Xyleborus vitiosus Schedl, 1940 & - & 1 & - & - & - \\
\hline $\begin{array}{c}\text { Xylosandrus retusus (Eichhoff, 1868) } \\
\text { FLEÓFAGAS }\end{array}$ & 210 & 761 & 863 & 73 & 1970 \\
\hline Cnesinus hispidus Eggers, 1943 & - & 1 & - & - & - \\
\hline Cnesinus sp.1 & 3 & 5 & 4 & - & - \\
\hline Cnesinus sp. 2 & - & 2 & - & - & - \\
\hline Cnesinus sp.3 & - & - & - & - & 1 \\
\hline Cnesinus sp.4 & 1 & - & - & - & - \\
\hline Hylocurus dimorphus (Schedl, 1939) & - & 2 & 1 & 1 & - \\
\hline Scolytus sp. 1 & - & 2 & - & - & - \\
\hline Scolytus sp. 2 & - & 1 & - & - & - \\
\hline $\mathrm{N}$ & 1116 & 1238 & 1300 & 315 & 3397 \\
\hline $\mathrm{S}$ & 31 & 53 & 40 & 38 & 35 \\
\hline $\mathrm{H}^{\prime}$ & 1,25 & 1,80 & 1,52 & 2,59 & 1,08 \\
\hline $\mathrm{E}$ & 0,36 & 0,45 & 0,41 & 0,71 & 0,30 \\
\hline
\end{tabular}

Em que: Número de indivíduos $(\mathrm{N})$, riqueza de espécies $(\mathrm{S})$, diversidade $\left(\mathrm{H}^{\prime}\right)$, equitabilidade $(\mathrm{E})$ e guilda dos espécimes de Scolytinae.

A maior diversidade de Scolytinae ocorreu na fazenda São Manoel, indicando que este ambiente é mais heterogêneo quanto à fauna destes insetos em relação aos demais locais (Tabela 2). $\mathrm{O}$ mesmo ocorrendo quanto à equitabilidade, demonstrando uma distribuição mais uniforme do número de indivíduos por espécies neste local. Segundo a hipótese da heterogeneidade do habitat, ambientes estruturalmente complexos provêm uma maior diversidade de nichos, possibilitando que os organismos explorem os recursos do ambiente de maneira diversificada, o que resulta no aumento da diversidade de espécies (TEWS, 2004).

Tais recursos ambientais podem estar representados pela variedade do material genético, visto que em São Manoel as armadilhas foram instaladas em três materiais genéticos distintos e em São Francisco em apenas uma espécie de eucalipto. Assim, os índices de diversidade que indicam a área São Manoel como um ambiente heterogêneo e com características próprias, possivelmente dependam, além da composição do florestamento, da composição da vegetação vizinha em que se encontra a assembleia de Scolytinae.

Todas as áreas estudadas possuem histórico similar de antropização, bem como de estrutura 
de sub-bosque, diferindo basicamente quanto à composiçao de espécies de eucalipto e tamanho da área plantada. O comportamento diferenciado da fauna de Scolytinae entre as localidades pode ser função de fatores associados ou não, como características fisiológicas e dendrológicas das espécies e híbridos de eucalipto.

O número de espécies coletado foi maior do que o encontrado em florestamento com Eucalyptus camaldulensis em Cuiabá - MS ( $\mathrm{S}=19)$ (ROCHA et al., 2011) e em Eucalyptus grandis $(\mathrm{S}=21)$ em Ilhota - SC (MÜLLER; ANDREIV, 2004). Com o mesmo modelo de armadilha de captura utilizada neste estudo, porém, em número superior (25) e com coletas semanais, Flechtmann, Ottati e Berisford (2001) capturaram 75 espécies, similar à quantidade aqui registrada, mas uma quantidade maior de besouros $(\mathrm{N}=12.335)$, em plantio de Eucalyptus grandis durante período de dois anos.

O maior número de besouros foi capturado na fazenda São Francisco $(46,1 \% ; \mathrm{N}=3397)$, seguida por Santa Rosa $(17,7 \% ; \mathrm{N}=1300)$, Cerro Alegre $(16,8 \% ; \mathrm{N}=1237)$ e Aroeira $(15,1 \% ; \mathrm{N}=1116)$ e a menor quantidade coletada foi em São Manoel $(4,3 \% ; \mathrm{N}=315)$ (Tabela 2). Embora o esforço amostral tenha sido o mesmo para todas as áreas, fatores como tipo de vegetação adjacente, estado fitossanitário da cultura, tamanho da área e variações climáticas podem ter sido responsáveis pela menor captura de Scolytinae na fazenda São Manoel.

O sul do Rio Grande do Sul encontra-se em uma região de transição entre os climas tropical e temperado (OVERBECK et al., 2009), o que pode explicar, em parte, a presença de espécies de Scolytinae tanto tropicais quanto temperadas. Entretanto, somente 24 espécimes de besouros fleófagos dos Scolytus, Cnesinus e Hylocurus foram capturados neste levantamento. Apesar de algumas espécies de fleófagos ocorrerem de forma abundante na região Sul do Brasil (FLECHTMANN; OTTATI; BERISFORD, 2001), a pouca quantidade coletada neste estudo provavelmente se deve ao tipo e à idade da vegetação em que foi realizada a amostragem, que não propicia locais de desenvolvimento de fleófagos, como espécies do gênero Scolytus que, em geral, preferem árvores de grande diâmetro (BRIGHT; STARK, 1973). Assim, os poucos exemplares coletados provavelmente vieram de áreas nativas vizinhas aos plantios de eucalipto, já que estes florestamentos se caracterizam pelo formato em mosaico, cujas áreas de proteção permanente, compostas basicamente por fragmentos de mata, banhados e capoeira, ocorrem juntas aos plantios.

Em todas as áreas amostradas, predominaram as espécies xilomicetófagas, que vivem em simbiose nutricional com fungos cultivados em túneis escavados no xilema da planta hospedeira (BIEDERMANN; KLEPZIG; TABORSKI, 2009) comprovando a importância desse grupo na região neotropical (ATKINSON; EQUIHUA-MARTINEZ, 1986; NOGUERA-MARTINEZ; ATKINSON, 1990; FLECHTMANN et al., 1995).

A tribo Corthylini, aqui representada pelos gêneros Corthylus, Monarthum, Amphicranus, Metacorthylus, Microcorthylus e Tricolus e a tribo Xyleborini, com os gêneros Ambrosiodmus, Dryocoetoides, Xyleborinus, Xyleborus e Xylosandrus foram as mais ocorrentes neste estudo, com 32 $(42,1 \%)$ e $24(31,6 \%)$ espécies, respectivamente (Tabela 2). Segundo Farrell et al. (2001) juntamente à subfamília Platyponinae, Corthylini e Xyleborini são essencialmente tropicais e compreendem $98 \%$ das 3.400 espécies descritas de besouros-da-ambrosia. Espécies da tribo Corthylini ocorrem em maior proporção na região sul do país, especialmente as dos gêneros Amphicranus, Corthylus, Corthylocurus, Monarthrum e Tricolus (FLECHTMANN; OTTATI; BERISFORD, 2001). Embora Corthylini tenha apresentado mais diversidade de gêneros, foi em Xyleborini que ocorreu a maior quantidade de indivíduos.

As outras tribos encontradas foram Cryphalini com 11 (14,5\%) espécies, Bothrosternini, cinco (6,6\%), Scolytini, duas (2,6\%), e Micracini e Ctenophorini, com uma (1,3\%) espécie.

Em todos os locais amostrados, Corthylus e Monarthum foram os gêneros com maior riqueza, com $13(17,1 \%)$ e $12(15,8 \%)$ espécies, embora tendo apresentado menor número de besouros $(9,7 \% ; \mathrm{N}=$ 715). As outras 51 (67,1\%) espécies distribuíram-se nos 16 gêneros restantes (Tabela 2). Escolitíneos do gênero Corthylus atacam galhos recém-quebrados, cortados ou pequenos caules de até $12 \mathrm{~cm}$ de diâmetro de vários hospedeiros, tais como espécies do gênero Cecropia, Acacia, Crotalaria, Theobroma cacao e algumas espécies de bambu (WOOD, 1982).

Do total de espécies coletadas, $82,9 \%$ ocorreu como dispersas, comuns e raras (Tabela 3). Esse padrão ocorre de forma frequente, independentemente do tipo de ecossistema (MAGURRAN, 2011) e, de acordo com Silveira Neto et al. (1976), locais em que os fatores limitantes e a competição interespecífica 
atuam intensamente, espécies mais comuns aumentam em número e espécies raras diminuem o seu nível populacional. Os autores também afirmam que, em uma assembleia, espécies raras ou aparentemente sem importância podem desempenhar funções indiretas importantes, assim como se adaptam a um novo meio e mantêm a vida da assembleia quando esta sofre alterações.

TABELA 3: Análise faunística de besouros Scolytinae capturados em cinco áreas florestadas com eucalipto no sul do Rio Grande do Sul, Brasil. Fevereiro de 2006 a outubro de 2010.

TABLE 3: Faunistic analysis of Scolytinae beetles caught in five forested areas with eucalypt in southern Rio Grande do Sul state, Brazil. February 2006 to October 2010.

\begin{tabular}{|c|c|c|c|c|c|c|c|c|c|c|c|c|c|c|c|c|c|c|c|c|}
\hline \multirow{2}{*}{ Espécies } & \multicolumn{4}{|c|}{ Abundância } & \multicolumn{5}{|c|}{ Constância } & \multicolumn{5}{|c|}{ Dominância } & \multicolumn{6}{|c|}{ Frequencia } \\
\hline & 1 & 2 & 3 & 4 & 5 & 1 & 2 & 3 & 4 & 5 & 1 & 2 & 3 & 4 & 5 & 1 & 2 & 3 & 4 & 5 \\
\hline XILOMICETÓFAGAS & & & & & & & & & & & & & & & & & & & & \\
\hline Ambrosiodmus hagedorni & $\mathrm{d}$ & - & - & - & $\mathrm{r}$ & $\mathrm{z}$ & - & - & - & $\mathrm{z}$ & $\mathrm{N}$ & - & - & - & $\mathrm{N}$ & $\mathrm{T}$ & - & - & - & $\mathrm{T}$ \\
\hline Ambrosiodmus obliquus & - & d & - & $\mathrm{c}$ & d & - & $\mathrm{z}$ & - & $\mathrm{y}$ & $\mathrm{z}$ & - & $\mathrm{N}$ & - & $\mathrm{N}$ & $\mathrm{N}$ & - & $\mathrm{T}$ & - & $\mathrm{F}$ & $\mathrm{T}$ \\
\hline Ambrosiodmus rusticus & - & - & - & - & $\mathrm{r}$ & - & - & - & - & $\mathrm{z}$ & - & - & - & - & $\mathrm{N}$ & - & - & - & - & $\mathrm{T}$ \\
\hline Ambrosiodmus sp. 1 & $\mathrm{~d}$ & d & $\mathrm{c}$ & $\mathrm{c}$ & $\mathrm{c}$ & z & z & $\mathrm{y}$ & $\mathrm{y}$ & $\mathrm{y}$ & $\mathrm{N}$ & $\mathrm{N}$ & $\mathrm{N}$ & $\mathrm{N}$ & $\mathrm{N}$ & $\mathrm{T}$ & $\mathrm{T}$ & $\mathrm{F}$ & $\mathrm{F}$ & $\mathrm{F}$ \\
\hline Ambrosiodmus sp. 2 & - & - & - & $\mathrm{d}$ & $\mathrm{r}$ & - & - & - & $\mathrm{z}$ & $\mathrm{z}$ & - & - & - & $\mathrm{N}$ & $\mathrm{N}$ & - & - & - & $\mathrm{T}$ & $\mathrm{T}$ \\
\hline Amphicranus sp.1 & $\mathrm{r}$ & $\mathrm{r}$ & - & - & $\mathrm{r}$ & $\mathrm{z}$ & $\mathrm{z}$ & - & - & z & $\mathrm{N}$ & $\mathrm{N}$ & - & - & $\mathrm{N}$ & $\mathrm{T}$ & $\mathrm{T}$ & - & - & $\mathrm{T}$ \\
\hline Amphicranus sp. 2 & $\mathrm{~d}$ & $\mathrm{r}$ & - & $\mathrm{d}$ & $\mathrm{r}$ & $\mathrm{z}$ & $\mathrm{z}$ & - & $\mathrm{z}$ & $\mathrm{z}$ & $\mathrm{N}$ & $\mathrm{N}$ & - & $\mathrm{N}$ & $\mathrm{N}$ & $\mathrm{T}$ & $\mathrm{T}$ & - & $\mathrm{T}$ & $\mathrm{T}$ \\
\hline Amphicranus sp. 3 & $\mathrm{~d}$ & - & - & - & - & z & - & - & - & - & $\mathrm{N}$ & - & - & - & - & $\mathrm{T}$ & - & - & - & - \\
\hline Coptoborus gracilens & - & $\mathrm{c}$ & - & - & - & - & $\mathrm{y}$ & - & - & - & - & $\mathrm{N}$ & - & - & - & - & $\mathrm{F}$ & - & - & - \\
\hline Corthylus antennarius & $\mathrm{m}$ & $\mathrm{m}$ & $\mathrm{m}$ & $\mathrm{m}$ & $\mathrm{m}$ & $\mathrm{w}$ & $\mathrm{w}$ & $\mathrm{w}$ & $\mathrm{w}$ & $\mathrm{w}$ & $\mathrm{D}$ & $\mathrm{D}$ & $\mathrm{D}$ & $\mathrm{D}$ & $\mathrm{D}$ & M & M & M & M & M \\
\hline Corthylus comitabilis & - & $\mathrm{d}$ & - & - & - & - & $\mathrm{z}$ & - & - & - & - & $\mathrm{N}$ & - & - & - & - & $\mathrm{T}$ & - & - & - \\
\hline Corthylus punctatus & $\mathrm{c}$ & $\mathrm{c}$ & $\mathrm{m}$ & $\mathrm{d}$ & $\mathrm{c}$ & $\mathrm{z}$ & $\mathrm{y}$ & $\mathrm{w}$ & $\mathrm{z}$ & $\mathrm{y}$ & $\mathrm{D}$ & $\mathrm{D}$ & $\mathrm{D}$ & $\mathrm{N}$ & $\mathrm{D}$ & $\mathrm{F}$ & $\mathrm{F}$ & M & $\mathrm{T}$ & $\mathrm{F}$ \\
\hline Corthylus sp. 1 & $\mathrm{c}$ & $\mathrm{r}$ & $\mathrm{d}$ & $\mathrm{c}$ & $\mathrm{r}$ & $\mathrm{y}$ & z & $\mathrm{y}$ & $\mathrm{y}$ & $\mathrm{z}$ & $\mathrm{D}$ & $\mathrm{N}$ & $\mathrm{N}$ & $\mathrm{N}$ & $\mathrm{N}$ & $\mathrm{F}$ & $\mathrm{T}$ & $\mathrm{T}$ & $\mathrm{F}$ & $\mathrm{T}$ \\
\hline Corthylus sp. 2 & $\mathrm{r}$ & $\mathrm{c}$ & $\mathrm{r}$ & - & $\mathrm{c}$ & $\mathrm{z}$ & $\mathrm{w}$ & $\mathrm{z}$ & - & $\mathrm{y}$ & $\mathrm{N}$ & $\mathrm{D}$ & $\mathrm{N}$ & - & $\mathrm{D}$ & $\mathrm{T}$ & $\mathrm{F}$ & $\mathrm{T}$ & - & $\mathrm{F}$ \\
\hline Corthylus sp. 3 & - & $\mathrm{r}$ & $\mathrm{r}$ & - & $\mathrm{r}$ & - & $\mathrm{z}$ & $\mathrm{z}$ & - & $\mathrm{z}$ & - & $\mathrm{N}$ & $\mathrm{N}$ & - & $\mathrm{N}$ & - & $\mathrm{T}$ & $\mathrm{T}$ & - & $\mathrm{T}$ \\
\hline Corthylus sp. 4 & $\mathrm{c}$ & $\mathrm{c}$ & $\mathrm{c}$ & $\mathrm{c}$ & d & $\mathrm{y}$ & $\mathrm{y}$ & $\mathrm{y}$ & z & $\mathrm{z}$ & $\mathrm{N}$ & $\mathrm{N}$ & $\mathrm{N}$ & $\mathrm{N}$ & $\mathrm{N}$ & $\mathrm{F}$ & F & F & $\mathrm{F}$ & $\mathrm{T}$ \\
\hline Corthylus sp. 5 & - & - & - & - & $\mathrm{r}$ & - & - & - & - & z & - & - & - & - & $\mathrm{N}$ & - & - & - & - & $\mathrm{T}$ \\
\hline Corthylus sp. 6 & - & - & - & $\mathrm{c}$ & $\mathrm{r}$ & - & - & - & z & z & - & - & - & $\mathrm{N}$ & $\mathrm{N}$ & - & - & - & $\mathrm{F}$ & $\mathrm{T}$ \\
\hline Corthylus sp. 7 & $\mathrm{r}$ & $\mathrm{c}$ & - & - & d & $\mathrm{z}$ & $\mathrm{w}$ & - & - & $\mathrm{z}$ & $\mathrm{N}$ & $\mathrm{D}$ & - & - & $\mathrm{N}$ & $\mathrm{T}$ & F & - & - & $\mathrm{T}$ \\
\hline Corthylus sp. 8 & - & - & $\mathrm{r}$ & $\mathrm{d}$ & - & - & - & $\mathrm{z}$ & z & - & - & - & $\mathrm{N}$ & $\mathrm{N}$ & - & - & - & $\mathrm{T}$ & $\mathrm{T}$ & - \\
\hline Corthylus sp. 9 & - & - & - & $\mathrm{d}$ & - & - & - & - & z & - & - & - & - & $\mathrm{N}$ & - & - & - & - & $\mathrm{T}$ & - \\
\hline Corthylus sp. 10 & - & $\mathrm{d}$ & - & - & - & - & $\mathrm{z}$ & - & - & - & - & $\mathrm{N}$ & - & - & - & - & $\mathrm{T}$ & - & - & - \\
\hline Cryptocarenus heveae & - & $\mathrm{r}$ & - & - & - & - & $\mathrm{z}$ & - & - & - & - & $\mathrm{N}$ & - & - & - & - & $\mathrm{T}$ & - & - & - \\
\hline Cryptocarenus brevicollis & $\mathrm{r}$ & $\mathrm{d}$ & - & - & - & z & $\mathrm{z}$ & - & - & - & $\mathrm{N}$ & $\mathrm{N}$ & - & - & - & $\mathrm{T}$ & $\mathrm{T}$ & - & - & - \\
\hline Cryptocarenus diadematus & - & $\mathrm{r}$ & $\mathrm{r}$ & - & $\mathrm{r}$ & - & z & $\mathrm{z}$ & - & $\mathrm{z}$ & - & $\mathrm{N}$ & $\mathrm{N}$ & - & $\mathrm{N}$ & - & $\mathrm{T}$ & $\mathrm{T}$ & - & $\mathrm{T}$ \\
\hline Cryptocarenus seriatus & - & $\mathrm{r}$ & $\mathrm{r}$ & - & d & - & $\mathrm{z}$ & $\mathrm{z}$ & - & $\mathrm{y}$ & - & $\mathrm{N}$ & $\mathrm{N}$ & - & $\mathrm{N}$ & - & $\mathrm{T}$ & $\mathrm{T}$ & - & $\mathrm{T}$ \\
\hline Cryptocarenus sp. 1 & - & $\mathrm{r}$ & - & - & - & - & $\mathrm{z}$ & - & - & - & - & $\mathrm{N}$ & - & - & - & - & $\mathrm{T}$ & - & - & - \\
\hline Dryocoetoides asperulus & - & $\mathrm{r}$ & - & - & - & - & $\mathrm{z}$ & - & - & - & - & $\mathrm{N}$ & - & - & - & - & $\mathrm{T}$ & - & - & - \\
\hline Hypothenemus dolosus & - & $\mathrm{r}$ & - & - & - & - & z & - & - & - & - & $\mathrm{N}$ & - & - & - & - & $\mathrm{T}$ & - & - & - \\
\hline Hypothenemus eruditus & $\mathrm{c}$ & - & $\mathrm{c}$ & - & $\mathrm{r}$ & z & - & $\mathrm{y}$ & - & z & $\mathrm{N}$ & - & $\mathrm{D}$ & - & $\mathrm{N}$ & $\mathrm{F}$ & - & $\mathrm{F}$ & - & $\mathrm{T}$ \\
\hline Hypothenemus sp. 1 & $\mathrm{~d}$ & - & d & - & - & $\mathrm{z}$ & - & $\mathrm{z}$ & - & - & $\mathrm{N}$ & - & $\mathrm{N}$ & - & - & $\mathrm{T}$ & - & $\mathrm{T}$ & - & - \\
\hline Hypothenemus sp. 2 & $\mathrm{~d}$ & - & $\mathrm{r}$ & $\mathrm{c}$ & - & $\mathrm{z}$ & - & z & $\mathrm{y}$ & - & $\mathrm{N}$ & - & $\mathrm{N}$ & $\mathrm{N}$ & - & $\mathrm{T}$ & - & $\mathrm{T}$ & $\mathrm{F}$ & - \\
\hline Hypothenemus sp. 3 & $\mathrm{c}$ & - & $\mathrm{r}$ & $\mathrm{d}$ & $\mathrm{r}$ & $\mathrm{z}$ & - & z & $\mathrm{z}$ & z & $\mathrm{D}$ & - & $\mathrm{N}$ & $\mathrm{N}$ & $\mathrm{N}$ & $\mathrm{F}$ & - & $\mathrm{T}$ & $\mathrm{T}$ & $\mathrm{T}$ \\
\hline Hypothenemus sp. 4 & - & - & $\mathrm{a}$ & - & - & - & - & $\mathrm{z}$ & - & - & - & - & $\mathrm{D}$ & - & - & - & - & M & - & - \\
\hline Metacorthylus sp. 1 & $\mathrm{c}$ & - & - & d & - & $\mathrm{z}$ & - & - & $\mathrm{z}$ & - & $\mathrm{N}$ & - & - & $\mathrm{N}$ & - & $\mathrm{F}$ & - & - & $\mathrm{T}$ & - \\
\hline Microcorthylus quadridens & $\mathrm{c}$ & $\mathrm{c}$ & $\mathrm{c}$ & $\mathrm{c}$ & d & $\mathrm{y}$ & $\mathrm{w}$ & $\mathrm{y}$ & $\mathrm{y}$ & $\mathrm{z}$ & $\mathrm{D}$ & $\mathrm{D}$ & $\mathrm{D}$ & $\mathrm{N}$ & $\mathrm{N}$ & $\mathrm{F}$ & $\mathrm{F}$ & $\mathrm{F}$ & $\mathrm{F}$ & $\mathrm{T}$ \\
\hline Microcorthylus sp.1 & $\mathrm{r}$ & - & - & d & - & $\mathrm{z}$ & - & - & z & - & $\mathrm{N}$ & - & - & $\mathrm{N}$ & - & $\mathrm{T}$ & - & - & $\mathrm{T}$ & - \\
\hline Monarthrum cristatum & - & $\mathrm{c}$ & - & - & $\mathrm{d}$ & - & $\mathrm{z}$ & - & - & $\mathrm{z}$ & - & $\mathrm{N}$ & - & - & $\mathrm{N}$ & - & $\mathrm{F}$ & - & - & $\mathrm{T}$ \\
\hline Monarthrum sp. 1 & $\mathrm{c}$ & - & $\mathrm{c}$ & $\mathrm{c}$ & $\mathrm{r}$ & $\mathrm{z}$ & - & $\mathrm{y}$ & $\mathrm{y}$ & $\mathrm{z}$ & $\mathrm{N}$ & - & $\mathrm{N}$ & $\mathrm{N}$ & $\mathrm{N}$ & $\mathrm{F}$ & - & $\mathrm{F}$ & $\mathrm{F}$ & $\mathrm{T}$ \\
\hline
\end{tabular}


TABELA 3: Continuação...

TABLE 3: Continued...

\begin{tabular}{|c|c|c|c|c|c|c|c|c|c|c|c|c|c|c|c|c|c|c|c|c|}
\hline \multirow{2}{*}{ Espécies } & \multicolumn{5}{|c|}{ Abundância } & \multicolumn{4}{|c|}{ Constância } & \multicolumn{6}{|c|}{ Dominância } & \multicolumn{5}{|c|}{ Frequência } \\
\hline & 1 & 2 & 3 & 4 & 5 & 1 & 2 & 3 & 4 & 5 & 1 & 2 & 3 & 4 & 5 & 1 & 2 & 3 & 4 & 5 \\
\hline Monarthrum sp. 2 & - & $\mathrm{r}$ & $\mathrm{r}$ & - & - & - & $\mathrm{z}$ & $\mathrm{z}$ & - & - & - & $\mathrm{N}$ & $\mathrm{N}$ & - & - & - & $\mathrm{T}$ & $\mathrm{T}$ & - & - \\
\hline Monarthrum sp. 3 & $\mathrm{~m}$ & $\mathrm{a}$ & $\mathrm{c}$ & $\mathrm{c}$ & $\mathrm{m}$ & $\mathrm{w}$ & $\mathrm{w}$ & $\mathrm{y}$ & $\mathrm{y}$ & $\mathrm{w}$ & $\mathrm{D}$ & $\mathrm{D}$ & $\mathrm{D}$ & $\mathrm{N}$ & $\mathrm{D}$ & M & M & $\mathrm{F}$ & $\mathrm{F}$ & M \\
\hline Monarthrum sp. 4 & $\mathrm{a}$ & $\mathrm{c}$ & $\mathrm{r}$ & $\mathrm{c}$ & $\mathrm{m}$ & $\mathrm{w}$ & $\mathrm{y}$ & $\mathrm{z}$ & $\mathrm{y}$ & $\mathrm{w}$ & $\mathrm{D}$ & $\mathrm{N}$ & $\mathrm{N}$ & $\mathrm{D}$ & $\mathrm{D}$ & M & $\mathrm{F}$ & $\mathrm{T}$ & $\mathrm{F}$ & M \\
\hline Monarthrum sp. 5 & $\mathrm{~m}$ & $\mathrm{a}$ & $\mathrm{r}$ & $\mathrm{c}$ & $\mathrm{m}$ & $\mathrm{w}$ & $\mathrm{w}$ & $\mathrm{z}$ & $\mathrm{y}$ & $\mathrm{w}$ & $\mathrm{D}$ & $\mathrm{D}$ & $\mathrm{N}$ & $\mathrm{N}$ & $\mathrm{D}$ & M & M & $\mathrm{T}$ & $\mathrm{F}$ & M \\
\hline Monarthrum sp. 6 & $\mathrm{~d}$ & - & - & d & $\mathrm{m}$ & $\mathrm{z}$ & - & - & $\mathrm{z}$ & $\mathrm{y}$ & $\mathrm{N}$ & - & - & $\mathrm{N}$ & $\mathrm{D}$ & $\mathrm{T}$ & - & - & $\mathrm{T}$ & M \\
\hline Monarthrum sp. 7 & $\mathrm{r}$ & - & $\mathrm{r}$ & - & $\mathrm{c}$ & $\mathrm{z}$ & - & $\mathrm{z}$ & - & $\mathrm{y}$ & $\mathrm{N}$ & - & $\mathrm{N}$ & - & $\mathrm{D}$ & $\mathrm{T}$ & - & $\mathrm{T}$ & - & $\mathrm{F}$ \\
\hline Monarthrum sp. 8 & $\mathrm{c}$ & - & $\mathrm{r}$ & - & $\mathrm{m}$ & $\mathrm{y}$ & - & $\mathrm{z}$ & - & $\mathrm{w}$ & $\mathrm{D}$ & - & $\mathrm{N}$ & - & $\mathrm{D}$ & $\mathrm{F}$ & - & $\mathrm{T}$ & - & M \\
\hline Monarthrum sp. 9 & $\mathrm{r}$ & d & - & - & $\mathrm{d}$ & $\mathrm{z}$ & $\mathrm{z}$ & - & - & $\mathrm{z}$ & $\mathrm{N}$ & $\mathrm{N}$ & - & - & $\mathrm{N}$ & $\mathrm{T}$ & $\mathrm{T}$ & - & - & $\mathrm{T}$ \\
\hline Monarthrum sp. 10 & d & $\mathrm{r}$ & - & - & d & $\mathrm{z}$ & $\mathrm{z}$ & - & - & $\mathrm{z}$ & $\mathrm{N}$ & $\mathrm{N}$ & - & - & $\mathrm{N}$ & $\mathrm{T}$ & $\mathrm{T}$ & - & - & $\mathrm{T}$ \\
\hline Monarthrum sp. 11 & $\mathrm{r}$ & - & - & - & $\mathrm{r}$ & $\mathrm{z}$ & - & - & - & $\mathrm{z}$ & $\mathrm{N}$ & - & - & - & $\mathrm{N}$ & $\mathrm{T}$ & - & - & - & $\mathrm{T}$ \\
\hline Scolytodes bolivianus & - & - & - & - & $\mathrm{r}$ & - & - & - & - & $\mathrm{z}$ & - & - & - & - & $\mathrm{N}$ & - & - & - & - & $\mathrm{T}$ \\
\hline Tricolus sp. 1 & $\mathrm{r}$ & - & $\mathrm{c}$ & - & $\mathrm{r}$ & $\mathrm{z}$ & - & $\mathrm{y}$ & - & $\mathrm{z}$ & $\mathrm{N}$ & - & $\mathrm{D}$ & - & $\mathrm{N}$ & $\mathrm{T}$ & - & $\mathrm{F}$ & - & $\mathrm{T}$ \\
\hline Xyleborinus gracilis & - & $\mathrm{c}$ & - & - & $\mathrm{c}$ & - & $\mathrm{z}$ & - & - & $\mathrm{z}$ & - & $\mathrm{N}$ & - & - & $\mathrm{N}$ & - & $\mathrm{F}$ & - & - & $\mathrm{F}$ \\
\hline Xyleborinus saxeseni & $\mathrm{m}$ & $\mathrm{m}$ & s & $\mathrm{s}$ & $\mathrm{m}$ & $\mathrm{w}$ & $\mathrm{w}$ & $\mathrm{w}$ & $\mathrm{w}$ & $\mathrm{y}$ & $\mathrm{D}$ & $\mathrm{D}$ & S & S & $\mathrm{D}$ & M & M & $\mathrm{U}$ & $\mathrm{U}$ & $\mathrm{F}$ \\
\hline Xyleborinus sentosus & $\mathrm{c}$ & $\mathrm{c}$ & $\mathrm{m}$ & $\mathrm{c}$ & $\mathrm{d}$ & $\mathrm{y}$ & $\mathrm{y}$ & $\mathrm{w}$ & $\mathrm{y}$ & $\mathrm{z}$ & $\mathrm{D}$ & $\mathrm{N}$ & $\mathrm{D}$ & $\mathrm{N}$ & $\mathrm{N}$ & $\mathrm{F}$ & $\mathrm{F}$ & M & $\mathrm{F}$ & $\mathrm{T}$ \\
\hline Xyleborinus sp. 1 & - & $\mathrm{c}$ & - & - & - & - & $\mathrm{y}$ & - & - & - & - & $\mathrm{D}$ & - & - & - & - & $\mathrm{F}$ & - & - & - \\
\hline Xyleborinus sp. 2 & $\mathrm{c}$ & $\mathrm{r}$ & $\mathrm{m}$ & $\mathrm{m}$ & $\mathrm{a}$ & $\mathrm{y}$ & $\mathrm{z}$ & $\mathrm{y}$ & $\mathrm{w}$ & $\mathrm{y}$ & $\mathrm{D}$ & $\mathrm{N}$ & $\mathrm{D}$ & $\mathrm{D}$ & $\mathrm{D}$ & $\mathrm{F}$ & $\mathrm{T}$ & M & M & M \\
\hline Xyleborinus sp. 3 & - & - & $\mathrm{c}$ & - & $\mathrm{r}$ & - & - & $\mathrm{z}$ & - & $\mathrm{z}$ & - & - & $\mathrm{D}$ & - & $\mathrm{N}$ & - & - & $\mathrm{F}$ & - & $\mathrm{T}$ \\
\hline Xyleborinus sp. 4 & - & - & - & - & $\mathrm{r}$ & - & - & - & - & $\mathrm{z}$ & - & - & - & - & $\mathrm{N}$ & - & - & - & - & $\mathrm{T}$ \\
\hline Xyleborinus sp. 5 & - & - & - & - & d & - & - & - & - & $\mathrm{z}$ & - & - & - & - & $\mathrm{N}$ & - & - & - & - & $\mathrm{T}$ \\
\hline Xyleborus affinis & $\mathrm{c}$ & - & - & d & $\mathrm{d}$ & $\mathrm{y}$ & - & - & $\mathrm{z}$ & $\mathrm{z}$ & $\mathrm{D}$ & - & - & $\mathrm{N}$ & $\mathrm{N}$ & $\mathrm{F}$ & - & - & $\mathrm{T}$ & $\mathrm{T}$ \\
\hline Xyleborus ferrugineus & $\mathrm{m}$ & $\mathrm{m}$ & $\mathrm{m}$ & $\mathrm{c}$ & $\mathrm{m}$ & $\mathrm{w}$ & $\mathrm{y}$ & $\mathrm{w}$ & $\mathrm{y}$ & $\mathrm{w}$ & $\mathrm{D}$ & $\mathrm{D}$ & $\mathrm{D}$ & $\mathrm{D}$ & $\mathrm{D}$ & M & M & M & $\mathrm{F}$ & M \\
\hline Xyleborus neivai & $\mathrm{d}$ & $\mathrm{c}$ & $\mathrm{r}$ & $\mathrm{c}$ & $\mathrm{c}$ & $\mathrm{z}$ & $\mathrm{y}$ & $\mathrm{z}$ & $\mathrm{y}$ & $\mathrm{y}$ & $\mathrm{N}$ & $\mathrm{N}$ & $\mathrm{N}$ & $\mathrm{N}$ & $\mathrm{D}$ & $\mathrm{T}$ & $\mathrm{F}$ & $\mathrm{T}$ & $\mathrm{F}$ & $\mathrm{F}$ \\
\hline Xyleborus reconditus & - & - & $\mathrm{r}$ & - & - & - & - & $\mathrm{z}$ & - & - & - & - & $\mathrm{N}$ & - & - & - & - & $\mathrm{T}$ & - & - \\
\hline Xyleborus sp. 1 & - & - & $\mathrm{r}$ & d & $\mathrm{r}$ & - & - & z & $\mathrm{z}$ & $\mathrm{z}$ & - & - & $\mathrm{N}$ & $\mathrm{N}$ & $\mathrm{N}$ & - & - & $\mathrm{T}$ & $\mathrm{T}$ & $\mathrm{T}$ \\
\hline Xyleborus sp. 2 & - & - & $\mathrm{c}$ & - & - & - & - & $\mathrm{z}$ & - & - & - & - & $\mathrm{D}$ & - & - & - & - & $\mathrm{F}$ & - & - \\
\hline Xyleborus sp. 3 & $\mathrm{r}$ & - & - & - & - & $\mathrm{z}$ & - & - & - & - & $\mathrm{N}$ & - & - & - & - & $\mathrm{T}$ & - & - & - & - \\
\hline Xyleborus vitiosus & - & - & - & - & $\mathrm{r}$ & - & - & - & - & $\mathrm{z}$ & - & - & - & - & $\mathrm{N}$ & - & - & - & - & $\mathrm{T}$ \\
\hline Xylosandrus retusus & $\mathrm{s}$ & $\mathrm{m}$ & $\mathrm{s}$ & $\mathrm{s}$ & $\mathrm{s}$ & $\mathrm{w}$ & $\mathrm{w}$ & $\mathrm{w}$ & $\mathrm{w}$ & $\mathrm{w}$ & $\mathrm{S}$ & $\mathrm{D}$ & $\mathrm{S}$ & $\mathrm{S}$ & $\mathrm{S}$ & $\mathrm{U}$ & M & $\mathrm{U}$ & $\mathrm{F}$ & $\mathrm{U}$ \\
\hline \multicolumn{21}{|l|}{ FLEÓFAGAS } \\
\hline Cnesinus hispidus & - & - & - & - & $\mathrm{r}$ & - & - & - & - & $\mathrm{z}$ & - & - & - & - & $\mathrm{N}$ & - & - & - & - & $\mathrm{T}$ \\
\hline Cnesinus sp. 1 & $\mathrm{c}$ & - & - & $\mathrm{c}$ & $\mathrm{c}$ & $\mathrm{z}$ & - & - & $\mathrm{z}$ & $\mathrm{z}$ & $\mathrm{N}$ & - & - & $\mathrm{N}$ & $\mathrm{N}$ & $\mathrm{F}$ & - & - & $\mathrm{F}$ & $\mathrm{F}$ \\
\hline Cnesinus sp. 2 & - & - & - & - & $\mathrm{r}$ & - & - & - & - & $\mathrm{z}$ & - & - & - & - & $\mathrm{N}$ & - & - & - & - & $\mathrm{T}$ \\
\hline Cnesinus sp. 3 & - & - & $\mathrm{r}$ & - & - & - & - & $\mathrm{z}$ & - & - & - & - & $\mathrm{N}$ & - & - & - & - & $\mathrm{T}$ & - & - \\
\hline Cnesinus sp. 4 & - & - & - & $\mathrm{d}$ & - & - & - & - & $\mathrm{z}$ & - & - & - & - & $\mathrm{N}$ & - & - & - & - & $\mathrm{T}$ & - \\
\hline Hylocurus dimorphus & $\mathrm{r}$ & $\mathrm{r}$ & - & - & $\mathrm{r}$ & z & z & - & - & $\mathrm{z}$ & $\mathrm{N}$ & $\mathrm{N}$ & - & - & $\mathrm{N}$ & $\mathrm{T}$ & $\mathrm{T}$ & - & - & $\mathrm{T}$ \\
\hline Scolytus sp. 1 & - & - & - & - & $\mathrm{r}$ & - & - & - & - & $\mathrm{z}$ & - & - & - & - & $\mathrm{N}$ & - & - & - & - & $\mathrm{T}$ \\
\hline Scolytus sp. 2 & - & - & - & - & $\mathrm{r}$ & - & - & - & - & $\mathrm{z}$ & - & - & - & - & $\mathrm{N}$ & - & - & - & - & $\mathrm{T}$ \\
\hline
\end{tabular}

Em que: 1: Arroio Grande - Santa Rosa; 2: São Manoel - Pinheiro Machado; 3: São Francisco - Pedras Altas; 4: Aroeira - Candiota; 5: Cerro Alegre - Piratini. Abundância: $\mathrm{s}=$ superabundante; $\mathrm{m}=$ muito abundante; a = abundante; $\mathrm{c}=$ comum; $\mathrm{d}=$ dispersa; $\mathrm{r}=$ rara; Constância: $\mathrm{w}=$ constante; $\mathrm{y}=$ acessória; $\mathrm{z}=$ acidental; Dominância: $\mathrm{S}=$ superdominante; $\mathrm{D}=$ dominante; $\mathrm{N}=$ não dominante; Frequência: $\mathrm{U}=$ superfrequente; $\mathrm{M}=$ muito frequente; $\mathrm{F}=$ frequente; $\mathrm{T}=$ pouco frequente.

As espécies Xyleborinus saxeseni e Xylosandrus retusus foram as mais abundantes nas cinco áreas avaliadas, perfazendo $80,6 \%(\mathrm{~N}=5.938)$ do total de exemplares coletados, seguido de Corthylus antennarius, que embora em menor quantidade $(4,2 \% ; \mathrm{N}=306)$, foi a terceira espécie mais capturada (Tabela 2).

Muito embora Xylosandrus retusus seja uma espécie comumente capturada em diferentes regiões do país e em diferentes biomas, ela não ocorre com a abundância com que foi encontrada neste levantamento. 
Mas esteve entre as espécies mais abundantes em plantações de Eucalyptus grandis e Pinus taeda em Telêmaco Borba - PR (FLECHTMANN; OTTATI; BERISFORD, 2001), bem como em floresta nativa em Itaara - RS (PELENTIR, 2007) e em povoamento de Acacia mearnsii, Butiá - RS (MURARI, 2005).

A espécie Xyleborinus saxeseni possui relatos de ataque em várias espécies de eucalipto, como Eucalyptus paniculata, Eucalyptus citriodora, Eucalyptus obliqua e Eucalyptus saligna (WALKER, 2006). No presente estudo, a maior abundância desse escolitíneo ocorreu na fazenda São Francisco, na qual todas as armadilhas foram instaladas em talhões compostos por Eucalyptus saligna. Também esteve entre as mais coletadas em povoamentos de Acacia mearnsii nos municípios de Butiá - RS (MURARI, 2005) e Santa Maria - RS (MACHADO et al., 2014). Essa espécie também se desenvolve em coníferas (ATKINSON; PECK, 1994), assim como em plantas de outros gêneros além de Acacia, como Betula, Camellia, Malus, Prunus, Populus, Quercus, Fagus, Ulmus e Weinmannia racemosa (BROCKERHOFF; KNÍZEK; BAIN, 2003).

O escolitíneo Xyleborus affinis foi encontrado em três locais, no entanto, sua abundância não foi expressiva. Na região Sul do Brasil, a espécie é relatada como pouco frequente (MARQUES, 1984; 1989), mas ocorre de forma muito abundante acima do Trópico de Capricórnio, assim como Xyleborus ferrugineus (FLECHTMANN et al., 1995).

As espécies Xyleborus affinis e Xyleborus ferrugineus são consideradas como os besouros-daambrosia mais agressivos em plantios de eucalipto no Brasil (FLECHTMANN, 2000). A espécie Xyleborus ferrugineus, embora apresentando pouca abundância $(\mathrm{N}=169)$ foi a quarta espécie mais capturada. Esse besouro é vetor primário do fungo patogênico Ceratocystis fimbriata (CIESLA, 2011) e ataca qualquer planta lenhosa com mais de $10 \mathrm{~cm}$ de diâmetro (WOOD, 1982). No Brasil, é o mais comum em pinhais e frequentemente observado atacando árvores caídas em Agudos - SP (FLECHTMANN; OTTATI; BERISFORD, 1999). Em plantios de Eucalyptus grandis, em Antônio Dias - MG, esteve entre as três espécies mais coletadas de Scolytinae (MORALES et al., 1999).

Com relação à constância, a maioria das espécies de Scolytinae $(81,6 \%)$ foi acessória e acidental, evidenciando que poucas estiveram presentes ao longo do tempo de amostragem (Tabela 3).

Quanto à frequência, as espécies Xyleborinus saxeseni e Xylosandrus retusus foram as que mais se destacaram neste levantamento e grande parte $(51,3 \%)$ ocorreu de forma esporádica. Em povoamento de acácia-negra - RS (MURARI, 2005) essas espécies também estiveram entre os besouros mais frequentes. Tais escolitíneos também se destacaram quanto à dominância, na qual foram superdominantes ou dominantes (Tabela 3).

\section{CONCLUSÕES}

Dos espécimes de Scolytinae coletados nos locais florestados com Eucalyptus spp., Corthylini e Xyleborini são as tribos dominantes, sendo Corthylini com a maior riqueza e Xyleborini com a maior abundância de indivíduos. As espécies predominantes são Xylosandrus retusus e Xyleborinus saxeseni. Algumas espécies prejudiciais às plantações de eucalipto (Xyleborus affinis e Xyleborus ferrugineus) foram encontradas nas áreas estudadas, porém, em pequeno número.

\section{AGRADECIMENTOS}

À Fibria Celulose S/A, pela execução logística deste estudo e à Silvia Tanabe, pela identificação dos Scolytinae.

\section{REFERÊNCIAS}

ASSOCIAÇÃO BRASILEIRA DE PRODUTORES DE FLORESTAS PLANTADAS. Anuário Estatístico da ABRAF 2013: Ano base 2012. Brasília: BRAF, 2013. Disponível em: <http://www.abraflor.org.br/ estatísticas.asp>. Acesso em: 30 abr. 2016.

ALTIERI, M. A.; LETOURNEAU, D. K. Vegetation diversity and insect pest outbreaks. CRC Critical Reviews in Plant Sciences, United Kingdom, v. 2, p. 131-169, 1984. 
ATKINSON, T. H.; EQUIHUA-MARTINEZ, A. Biology of bark and ambrosia beetles (Coleoptera: Scolytidae and Platypodidae) of a tropical rain forest in southeastern Mexico with an annotated checklist of species. Annals of the Entomological Society of America, México, v. 79, n. 3, p. 414-423, 1986.

ATKINSON, T. H.; FOLTZ, J. L.; CONNOR, M. D. Flight patterns of phoem and wood-boring Coleoptera (Scolytidae, Platypodidae, Curculionidae, Buprestidae, Cerambycidae) in north Florida slash pine plantation. Environmental Entomology, College Park, v. 17, n. 3, p. 259-265, 1988.

ATKINSON, T. H.; PECK, S. B. Annoted checklist of the bark and ambrosia beetles (Coleoptera: Platypodidae and Scolytidae) of tropical southern Florida. The Florida Entomologist, Florida, v. 77, n. 3, p. 313-329, 1994.

BEAVER, R. A. Bark and ambrosia beetles (Coleoptera: Scolytidae) newly recorded from Fiji, and their potential economic importance. South Pacific Journal of Natural Science, Fiji, v. 9, p. 64-70, 1987.

BERTI FILHO, E. Coleopteros de importância florestal: 1-Scolytidae. IPEF, Piracicaba, v. 19, p. 39-43, 1979.

BERTI FILHO, E.; FLECHTMANN, C. A. H. A model of ethanol trap to collect Scolytidae and Platypodidae (Insecta, Coleoptera). IPEF, Piracicaba, v. 34. p. 53-56, 1986.

BIEDERMANN, P. H. W; KLEPZIG, K. D.; TABORSKI, M. Fungus cultivation by ambrosia beetles: Behavior and laboratory breeding success in three Xyleborine species. Environmental Entomology, Lanham, v. 38, n. 4, p. 1096-1105, 2009.

BRASIL. Ministério do Meio Ambiente. Mapas de cobertura vegetal dos biomas brasileiros: relatório Bioma Pampa. Brasília: MMA, 2007. Disponível em: <www.mma.gov.br/potalbio>. Acesso em: 03 maio 2012.

BRIGHT, D. E.; STARK, R. W. The bark and ambrosia beetles of California. Coleoptera: Scolytidae and Platypodidae. Berkeley: University of California, 1973. 169 p.

BROCKERHOFF, E. G.; KNÍZEK, M.; BAIN, J. Checklist of indigenous and adventive bark and ambrosia beetles (Curculionidae: Scolytinae and Platypodinae) of New Zealand and interceptions of exotic species (1952-2000). New Zealand Entomologist, New Zealand, v. 26, p. 29-44, 2003.

CIESLA, W. Bark and ambrosia beetles. In: CIESLA, W. Forest Entomology: a global perspective. [s. 1: s. n.], 2011. p. 173-202. Disponível em: <http://books.google.com > ... > Zoology > Entomology $>$. Acesso em: 17 fev. 2012.

FARRELL, B. D. et al. The evolution of agriculture in beetles (Curculionidae: Scolytinae and Platypodinae). Evolution, Missouri, v. 55, p. 2011-2027, 2001.

FLECHTMANN, C. A. H. Scolytidae in pine plantations overviews and situation in Brazil. Série Técnica IPEF, Piracicaba, v. 13, n. 33, p. 49-56, 2000.

FLECHTMANN, C. A. H. et al. Scolytidae em reflorestamento com pinheiros tropicais. Piracicaba: Programa Cooperativo de Manejo de Pragas Florestais - IPEF, 1995. 201 p.

FLECHTMANN, C. A. H.; OTTATI, A. L. T.; BERISFORD, C. W. Ambrosia and bark beetles (Scolytidae: Coleoptera) in pine and eucalypt stands in southern Brazil. Forest Ecology and Management, Amsterdan, v. 142, n. 1/3, p. 183-191, 2001.

FLECHTMANN, C. A. H.; OTTATI, A. L. T.; BERISFORD, C. W. Attraction of ambrosia beetles (Coleoptera: Scolytidae) to different tropical pine species in Brazil. Environmental Entomology, Lanham, v. 28 , n. 4 , p. $649-658,1999$.

HARRINGTON, T. C. Ecology and Evolution of Mycophagous Bark Beetles and Their Fungal Partners. In: VEGA, F. E.; BLACKWELL, M. (Eds). Ecological and evolutionary advances in insect-fungal associations. United Kingdom: Oxford University, 2005. p. 257-291.

HULCR, J.; SMITH, S. Xyleborini ambrosia beetles: an identification tool to the world genera. 2010. Disponível em: <http://itp.lucidcentral.org/id/wbb/xyleborini/index.htm>. Acesso em: 30 jul. 2012.

KOTTEK, M. World Map of the Köppen-Geiger climate classification updated. Meteorologische Zeitschrift, Stuttgart, v. 15, n. 3, p. 259-263, 2006.

MACHADO, L. et al. Escolitíneos associados a uma população de acácia-negra (Acacia mearnsii De Wild). Biotemas, Florianópolis, v. 27, n. 3, p. 57-63, 2014.

MAGURRAN, A. E. Medindo a diversidade ecológica. Curitiba: Ed. da UFPR, 2011. 261 p.

MARQUES, E. N. Índices faunísticos e grau de infestação por Scolytidae em madeira de Pinus spp. 
1989. 103 f. Tese (Doutorado em Ciências Florestais) - Curso de Pós-Graduação em Engenharia Florestal, Universidade Federal do Paraná, Curitiba, 1989.

MARQUES, E. N. Scolytidae e Platypodidae em Pinus taeda. 1984. 65 f. Dissertação (Mestrado em Ciências Florestais) - Universidade Federal do Paraná, Curitiba, 1984.

MORAES, J. C. et al. Effect of Eucalyptus species and soil type on infestation levels of heartwood termites (Insecta: Isoptera) in reforested areas of Brazil. Sociobiology, Chico, v. 39, p. 145-153, 2002.

MORAES, R. C. B. et al. Software para análise faunística - ANAFAU. In: SIMPÓSIO DE CONTROLE BIOLÓGICO, 2003, São Pedro, SP. Resumos... Piracicaba: ESALQ; USP, 2003. p. 195.

MORALES, N. E. et al. Índices populacionais de besouros Scolytidae em reflorestamento de Eucalyptus grandis W. Hill ex Maiden no Município de Antônio Dias, Minas Gerais. Revista Árvore, Viçosa, MG, v. 23, p. 359-363, 1999.

MÜLLER, J. A.; ANDREIV, J. Caracterização da família Scolytidae (Insecta: coleoptera) em três ambientes florestais. Revista Cerne, Lavras, v. 10, n. 1, p. 39-45, 2004.

MURARI, A. B. Levantamento populacional de Scolytidae (Coleoptera) em povoamento de Acácianegra (Acacia mearnsii De Wild). 2005. 64 f. Dissertação (Mestrado em Engenharia Florestal) Universidade Federal de Santa Maria, Santa Maria, 2005.

NOGUERA-MARTINEZ, F. A.; ATKINSON, T. H. Biogeography and biology of bark and ambrosia beetles (Coleoptera: Scolytidae and Platypodidae) of a mesicmontane forest in Mexico, with an annotated checklist of species. Annals of the Entomological Society of America, Washington, v. 83, n. 3, p. 453-466, 1990.

OKLAND, B. et al. Inter-species interactions and ecosystem effects of non-indigenous invasive and native tree-killing bark beetles. Biological Invasions, Netherlands, v. 13, p. 1151-1164, 2011.

OLIVEIRA, C. M.; FLECHTMANN, C. A. H.; FRIZZAS, M. R. First record of Xylosandrus compactus (Eichhoff) (Coleoptera: Curculionidae: Scolytinae) on soursop, Annona muricata L. (Annonaceae) in Brazil, with a list of host plants. Coleopterists Bulletin, Washington, v. 62, n. 1, p. 45-48, 2008.

OVERBECK, G. E. et al. Os Campos Sulinos: um bioma negligenciado. In: PLLAR, V. P. et al. (Eds.). Campos Sulinos: conservação e uso sustentável da biodiversidade. Brasília: MMA, 2009. p. 26-41.

PELENTIR, S. C. S. Eficiência de cinco modelos de armadilhas etanólicas na coleta de Coleoptera: Scolytidae, em floresta nativa no município de Itaara RS. 2007. 74 f. Dissertação (Mestrado em Engenharia Florestal) - Universidade Federal de Santa Maria, Santa Maria, 2007.

ROCHA, J. R. M. et al. Análise da Ocorrência de Coleópteros em Plantios de Eucalyptus camaldulensis Dehn. em Cuiabá, MT. Floresta e Ambiente, Seropédica, v. 18, n. 4, p. 343-352, 2011.

SILVA, M. D. Os cultivos florestais do pampa, no sul do Rio Grande do Sul: desafios, perdas e perspectivas frente ao avanço de novas fronteiras agrícolas. Floresta, Curitiba, v. 42, n. 1, p. 215-226, 2012.

SILVEIRA NETO, S. et al. Manual de ecologia dos insetos. São Paulo: Ceres, 1976. 419 p.

TEWS J. Animal species diversity driven by habitat heterogeneity/diversity: the importance of keystone structures. Journal of Biogeography, Oxford, v. 31, p. 79-92, 2004.

TURNBOUW, J. R.; FRANKLIN, R. T. Flight activity by Scolytidae in the northeast Georgia Piedmont (Coleoptera). Journal of the Georgia Entomological Society, Athens, v. 15, n. 1, p. 26-37, 1980.

WALKER, K. Fruit-tree pinhole borer (Xileborinus saxeseni). 2006. Disponível em: <http://dev.padil. gov.au:80/pests-and-diseases/Pest/Main/135733>. Acesso em: 12 ago. 2012.

WOOD, S. L. The bark and ambrosia beetles of North and Central America (Coleoptera: Scolytidae), a taxonomic monograph. Great Basin Naturalist Memoirs, n. 6, p. 1-1359, 1982. 